\title{
On Some Properties of the Output of a Pulsed Digital Oscillator Working with Multiple Resonances
}

\author{
Elena Blokhina and Orla Feely \\ School of Electrical, Electronic and Mechanical Engineering \\ University College Dublin \\ Dublin, Ireland
}

\author{
Jordi Ricart and Manuel Domínguez \\ Micro and Nano Technologies group \\ Universitat Politècnica de Catalunya \\ Barcelona, Spain
}

\begin{abstract}
In this paper, we study the possible output of the pulsed digital oscillator (PDO) with multiple resonant modes of the mechanical resonator in the feedback loop. PDOs are simple circuits that allow linear resonators to maintain self-sustained oscillations and can work as mass-change resonant sensors. For a resonant sensor, activation of higher vibration modes of a mechanical resonator can be a way to improve its performance. We show that the location of the sensing/actuation system affects the output and can enhance higher mechanical modes.
\end{abstract}

\section{INTRODUCTION}

Most of the methods that have been proposed to improve the sensitivity of gravimetric resonant sensors are based on increasing their operating frequency. For instance, parametric resonance amplification is an example of an efficient technique [1] that allows the excitation of the same mechanical structure at a higher frequency to improve sensitivity. The use of higher order modes generally serves to increase the sensitivity to mass changes, whereas it is desired in gyroscopes to avoid the activation of certain resonant modes of the inertial mass [2]. Depending on the specific application, the selective activation of different spatial vibration modes of a mechanical resonator can be one way to improve performance for a large number of MEMS sensors [3], [4].

The aim of this paper is to examine the output of the PDO [5]-[7]. working with multiple resonances of the mechanical structure (see fig. 1). Recently, we have shown that this topology is capable of actuating various spatial modes of the mechanical structure, depending on the sampling frequency and the number of delays in the feedback loop [8] and presented experimental results in [9]. In this work, we focus on the particular problem of how the location of the sensing/actuation system affects the output of the PDO. We show the plane spanned by the control parameters of the system illustrating excitation of various longitudinal vibrational modes (with higher frequencies) of the mechanical resonator in the feedback loop.

\section{StATEMENT OF THE PROBLEM}

Let us summarise below the statement of the problem [8] for convenience of readers. Later, in Sec. III we will proceed to new results of numerical simulations. We assume that the MEMS system we study utilises a clamped-free cantilever. We

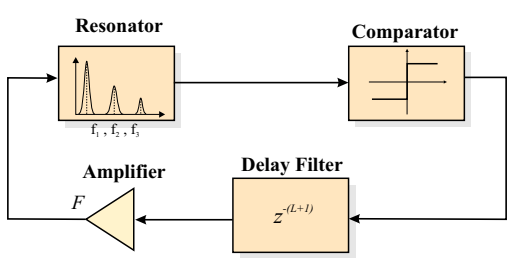

Fig. 1. The PDO general single-feedback topology consists of a resonator, a 1-bit quantizer (sign function), and a simple delay feedback filter, $G(z)=$ $z^{-(L+1)}$ (in the paper, $L=0$ ) and the feedback gain (in the paper $\sigma=+1$ ).

consider that the beam is excited by the external force $F(\xi, \tau)$. The equation and the boundary conditions that describe the transverse vibrations of the beam in dimensionless form are as follows [10]:

$$
\frac{\partial^{4} u}{\partial \xi^{4}}+\frac{\partial^{2} u}{\partial \tau^{2}}+\gamma \frac{\partial u}{\partial \tau}=F(\xi, \tau)
$$

$u(0, \tau)=\partial u(0, \tau) / \partial \xi=0$ and $\partial^{2} u(1, \tau) / \partial \xi^{2}=$ $\partial^{3} u(1, \tau) / \partial \xi^{3}=0$, with the dimensionless variables and parameters $\xi=x / l, \tau=\left(t / l^{2}\right) \sqrt{E I / \rho A}$ and $\gamma=c l^{2} / \sqrt{\rho A E I}$.

In (1), $u(x, t)$ is the transverse displacement at the position $\xi$ and time $\tau, E$ is the Young's modulus, $I$ is the moment of inertia of the cross-section (for beams with a rectangular cross-section $\left.I=b h^{3} / 12\right), \rho$ is the density, $A$ is the area of the cross-section, $c$ is the dissipation coefficient, $\gamma$ is the dimensionless dissipation parameter, $l$ is the length of the beam, $b$ is its width and $h$ is its thickness.

Next, we discretise the continuous equation (1) by using a truncated set of linear mode shapes [10], [11]

$$
u(\xi, \tau)=\sum_{i=1}^{M} \xi_{i}(\tau) \psi_{i}(\xi) .
$$

For a clamped-free beam, these functions are $\psi_{i}(\xi)=$ $\cosh \left(\Omega_{i} \xi\right)-\cos \left(\Omega_{i} \xi\right)+\nu_{i}\left(\sin \left(\Omega_{i} \xi\right)-\sinh \left(\Omega_{i} \xi\right)\right)$, where $\nu_{i}=$ $\left(\cos \Omega_{i}+\cosh \Omega_{i}\right) /\left(\sin \Omega_{i}+\sinh \Omega_{i}\right)$, and $\Omega_{i}$ are the roots of the equation $\cos \Omega \cosh \Omega=-1$.

Substituting (2) into (1), multiplying the latter equation by $\psi_{j}$, integrating over the beam domain and taking into account the orthogonality of the functions $\psi_{j}$, we obtain a system of ordinary differential equations with the functions 
$F_{i}(\tau)=\int_{0}^{1} \psi_{i}(\xi) F(\xi, \tau) d \xi$ in the right hand of the equations. These functions are the 'projections' of the external force to each eigenmode and define what quantity of the force is transmitted to excite the oscillations of a mode.

The external driving for the PDO has the following form:

$F(\xi, \tau)=-\sigma F_{0} \sum_{n} \operatorname{sign}\left(u\left(\xi_{s}, \tau_{n-L-1}\right)\right) \delta\left(\xi-\xi_{a}\right) \delta\left(\tau-\tau_{n}\right)$,

where $L+1$ is the number of delays in the feedback, $\tau_{n}=n T_{s}$ is the instants of time at which we apply the force pulse, $T_{s}=$ $1 / f_{s}$ are the dimensionless time between two impulses, $\delta(x)$ is the Dirac delta function and $\operatorname{sign}(x)$ is the signum function. In eq. (3), $\xi_{s}$ is the point of the microcantilever at which the sensing system measures the resonator position and $\xi_{a}$ is the point at which the actuating system applies the external driving in order to maintain the oscillations.

Thus the system that represents each spatial resonant mode in the PDO as the mass-spring-damper system with appropriate parameters such as the dissipation parameter $\gamma$ and the temporal frequency $\omega_{0 i}=\Omega_{i}^{2}\left(\Omega_{i}\right.$ are solutions of the corresponding equation) has the following form:

$$
\ddot{x}_{i}+\gamma \dot{x}_{i}+\Omega_{i}^{4} x_{i}=-\sigma F_{0 i} \sum_{n} b_{n-L-1} \delta\left(\tau-\tau_{n}\right),
$$

where $F_{0 i}=F_{0} \psi\left(\xi_{a}\right)$. Based on eq. (2) and on condition that $\psi_{1}\left(\xi_{s}\right)>0$, one can write that

$$
b_{n}=\operatorname{sign}\left\{x_{1}\left(\tau_{n}\right)+\beta_{2} x_{2}\left(\tau_{n}\right)+\ldots+\beta_{M} x_{M}\left(\tau_{n}\right)\right\},
$$

where

$$
\beta_{i}=\psi_{i}\left(\xi_{s}\right) / \psi_{1}\left(\xi_{s}\right) .
$$

Formula (6) introduces a family of parameters in order to avoid always operating in terms of the spatial functions $\psi_{i}$. The parameters $\beta_{i}$ now defines the "geometry" of the sensing system and the contribution of the $i$ th spatial mode to the overall position of the resonator.

In the same manner, we can introduce the parameters $\mu_{i}$ that defines the "geometry" of the actuating system and the impact of the external driving on the $i$ th mode excitation:

$$
\mu_{i}=\psi_{i}\left(\xi_{a}\right) / \psi_{1}\left(\xi_{a}\right), \quad F_{0 i}=\mu_{i} F_{01} .
$$

The examined system is subjected to pulsed excitation. If we define the sequence $\left\{x_{i, n}, \dot{x}_{i, n}\right\}=\left\{x_{i}\left(\tau_{n}\right), d x_{i}\left(\tau_{n}\right) / d \tau\right\}$, that is the sequence of the sampled 'position' and 'velocity' taken from the mass-spring-damper equations (4) for each $i$ th spatial mode, we will be able to formulate iterative equations that describe the dynamics of the system in terms of the introduced variables.

The set of iterative equations has the following form:

$$
\left(\begin{array}{c}
x_{i, n+1} \\
v_{i, n+1}
\end{array}\right)=\alpha_{i} \mathbf{R}\left(2 \pi f_{i}\right)\left(\begin{array}{c}
x_{i, n} \\
v_{i, n}
\end{array}\right)+\left(\begin{array}{c}
0 \\
\zeta_{i}
\end{array}\right) b_{n-L},
$$

where $v_{i, n}=-\left(\rho_{i} x_{i, n}+\dot{x}_{i, n} / \Omega_{i}^{2}\right) /\left(\sqrt{1-\rho_{i}^{2}}\right)$ and $R(\theta)=$ $\left(\begin{array}{c}\cos \theta-\sin \theta \\ \sin \theta \cos \theta\end{array}\right)$. In the set (8), we introduced the following dimensionless parameters: the dimensionless damping parameter $\rho_{i}=\gamma /\left(2 \Omega_{i}^{2}\right)$, is the normalised frequency (or normalised sample ratio in terms of the paper [12])

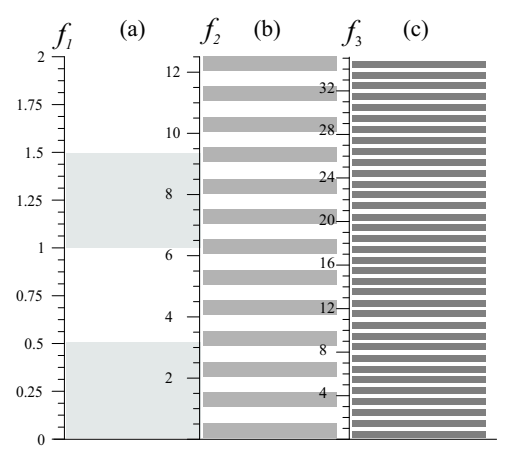

Fig. 2. Regions of the normalised frequencies $f_{1,2,3}$ that correspond to the oscillation and anti-oscillation regimes of the first (a), the second (b) and the third longitudinal spatial modes. Anti-oscillation regions are shown by the white colour.

$f_{i}=\left(\Omega_{i}^{2} / f_{s}\right) \sqrt{1-\rho_{i}^{2}}$, the normalised increment (note that it depends on the sign of the feedback loop $\sigma$ ) $\zeta_{i}=\left(\sigma F_{0 i}\right) /\left(\Omega_{i}^{2} \sqrt{1-\rho_{i}^{2}}\right)$ and the contraction factor $\alpha_{i}=$ $\exp \left(\left(-2 \pi \rho_{i} f_{i}\right) /\left(\sqrt{\left.1-\rho_{i}^{2}\right)}\right)\right.$. In (8), the first letter of the index, i.e. ' $i$ ', refers to the number of a spatial mode, and the second letter, i.e. ' $n$ ', refers to the iteration number.

\section{Numerical Simulations}

\section{A. Oscillations and Anti-oscillations}

Let us consider the first three longitudinal spatial modes with the lowest frequencies: $\Omega_{1}=1.8751, \Omega_{2}=2.69409$ and $\Omega_{3}=7.8547$. Evolution of each mode is defined by the iterative equation (8) with appropriate parameters. The overall position of the resonator is described as $u\left(\xi_{s}, \tau_{n}\right)=$ $\psi_{1}\left(\xi_{s}\right)\left\{x_{n, 1}+\beta_{2} x_{n, 2}+\beta_{3} x_{n, 3}\right\}$.

In [8], [13], the dynamics of the PDO with one resonance and multiple delays in the feedback loop has been considered. We observed the normal oscillation mode (large amplitude oscillations) and anti-oscillation mode (very small amplitude

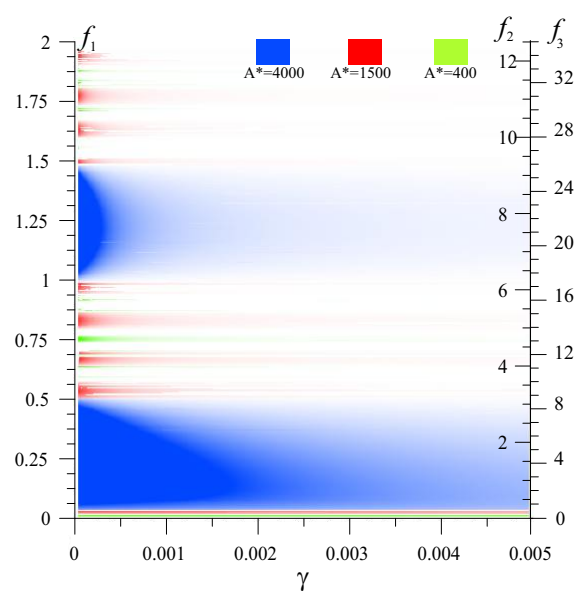

Fig. 3. Plane of parameters $\left(\gamma, f_{1}\right)$ - the dissipation parameter and the sampling ratio in which shades of different colours show the intensity of oscillations of different spatial modes. The blue colour shows the amplitude of oscillations of the first spatial mode, the red colour shows the second mode and the green colour - the third mode. The actuating and sensing structures are placed at $\xi_{s}=\xi_{a}=1$. 
oscillations in terms of [8]) depending on values of the normalised frequency, the sign of the feedback loop and the number of delays.

Let us introduce now oscillations and anti-oscillations for the set of equations (8). The basic parameters in our problem are the parameters that relate to the the first (fundamental) mode. Using them, we can express parameters for the higher order modes (the second, etc.). Figure 2 shows oscillations and anti-oscillations for all three modes where oscillations are shown by the grey colours and anti-oscillations by blank areas.

\section{B. Planes of parameters}

Extensive numerical simulations have shown that the output of the system displays oscillations that can be associated with only one of spatial modes while the other mode will be "suppressed". For instance, where the amplitude of the first mode is large, the amplitudes of the second and third mode are very small.

To illustrate the dynamics of the system, we consider the plane spanned by the control parameters - dissipation $\gamma$ and the normalised frequency $f_{1}$. Figure 3 represents distributions of the amplitude of the three spatial modes in different colours in that plane. Firstly, it is defined which mode the output corresponds to (the "dominant" mode with the largest amplitude) at the given $\gamma$ and $f_{1}$. Secondly, we represent the amplitude of this mode using a shade of the chosen colour (shades of the blue colour show the first longitudinal spatial mode, shades of the red show the second and shades of the green the third mode). The higher the amplitude of oscillations, the darker the shade of the colour that represents the corresponding point in the plane. Note that all amplitudes that are more than $A^{*}$ (see the figure) will be represented with the same shade. The plane has been calculated with the zero initial conditions $x_{0, i}=y_{0, i}=0$ and at the position of sensing/actuating system at $\xi_{s}=\xi_{a}=1$.

In the above case, the position of the sensing/actuating system is chosen in such a way that the contribution of all modes in the sequence of signs (5) is of the same order since $\beta_{2}=-1$ and $\beta_{3}=+1$. However, the portions of the external driving differ due to the ratio of the frequencies: $\zeta_{2} \approx \mu_{2}\left(\Omega_{1} / \Omega_{2}\right)^{2} \zeta_{1}$ and $\zeta_{3} \approx \mu_{3}\left(\Omega_{1} / \Omega_{3}\right)^{2} \zeta_{1}$. Therefore, higher modes (the second and the third) receive lower portions of external driving and the first mode dominates over all regions of its oscillations. In this case, the PDO displays the second or third modes strictly on condition that the first mode is in anti-oscillations.

Since the ratios $\left(\Omega_{1} / \Omega_{2}\right)^{2}$ and $\left(\Omega_{1} / \Omega_{3}\right)^{2}$ are fixed, it is natural to consider different $\mu_{2}$ and $\mu_{3}$ in order to enhance higher modes and to suppress the fundamental one, i.e., to consider other positions of the sensing/actuating system. The next example is shown in fig. 5 at $\xi_{s}=\xi_{a}=0.5$. At this location of the sensing system, the function $\psi_{2}(\xi)$ has a maximum, $\psi_{1}(\xi)$ has a small value and $\psi_{3}(\xi)$ is close to zero. As can be seen from the figure, oscillations corresponding to the second mode are significant at some value of $f_{1}$ where in the previous case we observed oscillations of only the first mode. In addition, the bands showing oscillations of the second are more defined and intense compared to fig. 3.

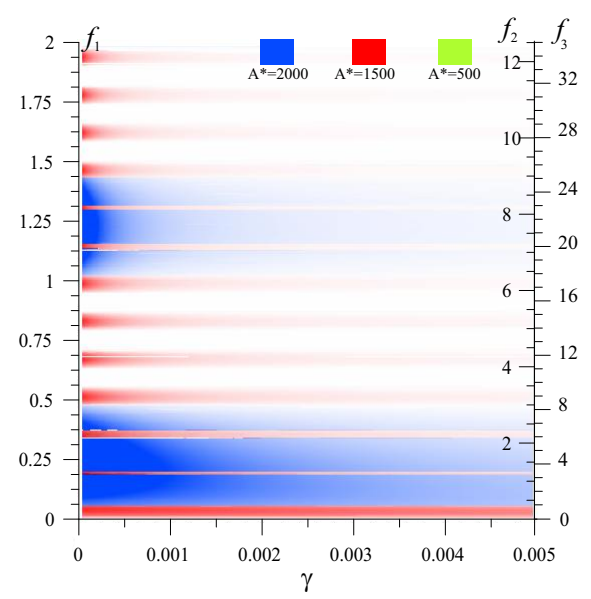

Fig. 4. Plane of parameters $\left(\gamma, f_{1}\right)$ - the dissipation parameter and the sampling ratio in which shades of different colours show the intensity of oscillations of different spatial modes. The blue colour shows the amplitude of oscillations of the first spatial mode, the red colour shows the second mode and the green colour - the third mode. The actuating and sensing structures are placed at $\xi_{s}=\xi_{a}=0.5$.

In order to obtain more intense oscillation of the third mode, we consider the case $\xi_{s}=\xi_{a}=0.3$, i.e. the sensing system is located at the maximum of $\psi_{3}(\xi)$. The amplitudes of oscillations of all modes are significantly smaller, however, one can see many regions of $f_{1}$ where the PDO demonstrates the output that correspond to the second and the third modes.

\section{C. "Multistability" of the modes in the PDO.}

Figure 2 shows that at the same value of the normalised frequency $f_{1}$ there can be simultaneous oscillations of multiple spatial modes. In the case when two or more modes are in oscillation regimes, the output of the PDO depends on the initial conditions (initial deformation of the cantilever). The arbitrary initial deformation can be presented in the form

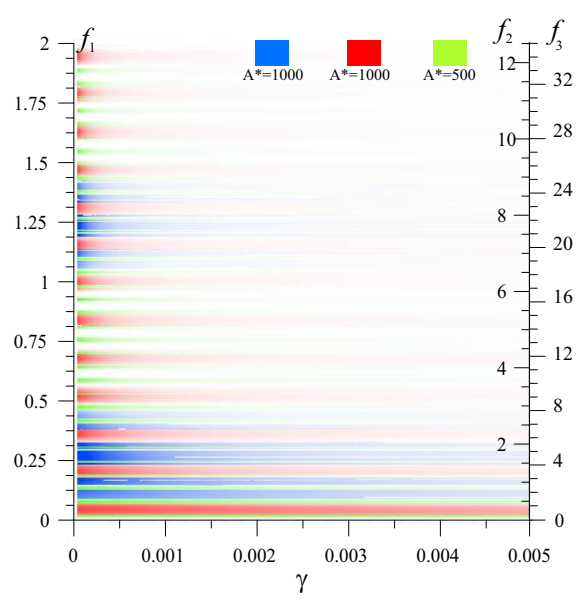

Fig. 5. Plane of parameters $\left(\gamma, f_{1}\right)$ - the dissipation parameter and the sampling ratio in which shades of different colours show the intensity of oscillations of different spatial modes. The blue colour shows the amplitude of oscillations of the first spatial mode, the red colour shows the second mode and the green colour — the third mode. The actuating and sensing structures are placed at $\xi_{s}=\xi_{a}=0.3$. 

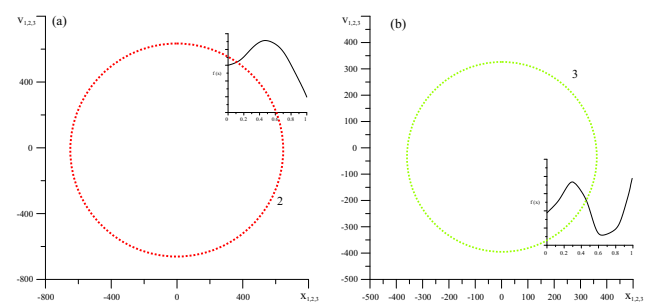

Fig. 6. The output of the resonator, $\gamma=0.001, f_{1}=0.1845, \xi_{s}=\xi_{a}=$ 0.3 , (a) - at the initial conditions $x_{0}^{1}=x_{0}^{2}=x_{0}^{3}=0$; (b) - at the initial conditions $x_{0}^{1}=80, x_{0}^{2}=20, x_{0}^{3}=0$.

$u(\xi, 0)=x_{0}^{(1)} \psi_{1}(\xi)+x_{0}^{(2)} \psi_{2}(\xi)+x_{0}^{(3)} \psi_{3}(\xi)$ where $x_{0}^{(i)}$ are considered as the initial conditions for maps 8. Figure 6 illustrates that the output mode and the cantilever profile depends of $x_{0}^{(i)}$.

We choose the normalised frequency value $f_{1}=0.1845$ at which all three modes are in an oscillation region. Figure 7 shows the plane spanned by the initial conditions $\left(x_{0}^{(1)}, x_{0}^{(2)}\right)$ and the the fixed value of $x_{0}^{(3)}=0, f_{1}=0.1845, \gamma=0.001$ and $\xi_{a}=\xi_{a}=0.3$. Note that the scale of the axes corresponds to very small initial deformations of the beam (typically, the amplitudes of oscillations $\sim 1000$ ). In the figure, the blank area corresponds to the second mode of the PDO output. The very small grey region around the origin corresponds to the third mode (this mode one observes if zero initial conditions are chosen, for instance, as for the planes 3-4). Insets in the figure show typical deformations of the cantilever which lead to a particular oscillations. Figure 8 is similar to plot 7 but calculated at $x_{0}^{(3)}=10$, i.e., with a small components of the third mode in the initial deflection of the beam.

\section{CONCLUSIONS}

The PDO is capable of selective excitation of various spatial modes of the mechanical structure in the feedback loop. We have shown that besides such control parameters as the

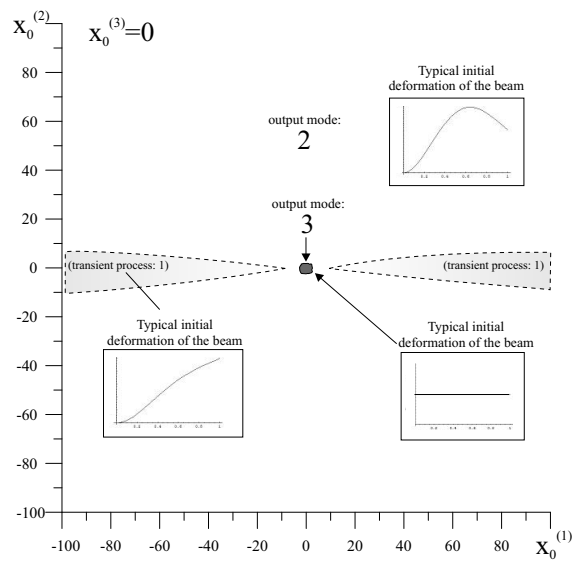

Fig. 7. Plane of initial conditions $\left(x_{0}^{1}, x_{0}^{2}\right)$ showing the possible output of the resonator. $\gamma=0.001, f_{1}=0.1845, \xi_{s}=\xi_{a}=0.3$. The gray areas outlined by dashed lines are the areas with extremely long transient processes (up to $10^{5}$ iterations) when the system displays first oscillations of the first spatial mode and converge eventually to oscillations that correspond to the third mode.

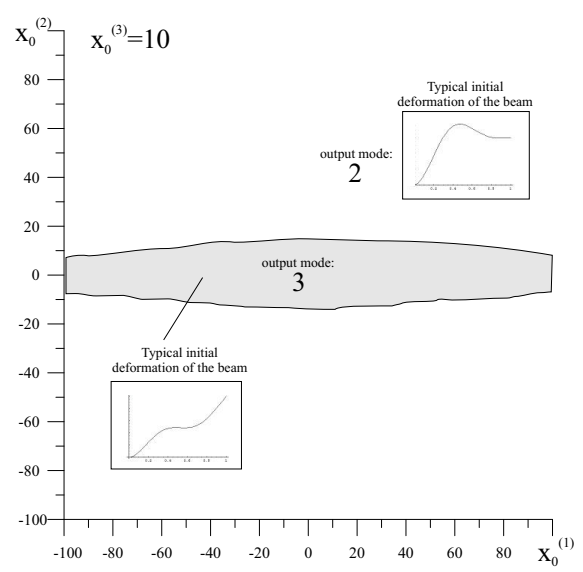

Fig. 8. Plane of initial conditions $\left(x_{0}^{1}, x_{0}^{2}\right)$ showing the possible output of the resonator. $\gamma=0.001, f_{1}=0.1845, \xi_{s}=\xi_{a}=0.3$.

normalized frequency and the number of delays, the output of the PDO depends on the position of the sensing/actuating system. The location of the latter system may enhance highorder modes and improve the sensitivity of the device. In the case when the sampling frequency is chosen in such a way that two or more modes are in oscillations, the output of the circuit depends on initial conditions, i.e., on the initial deformation of the beam.

\section{REFERENCES}

[1] W. Zhang and K. L. Turner, "Application of parametric resonance amplification in a single-crystal silicon micro-oscillator based mass sensor," Sensors and Actuators A, vol. 122, pp. 23-30, 2005.

[2] C. Acar and A. M. Shkel, "Structural design and experimental characterization of torsional micromachined gyroscopes with non-resonant drive mode," J. Micromechan. Microeng., vol. 14, pp. 15-25, 2004.

[3] S. Dohn, R. Sandberg, W. Svendsen, and A. Boisen, "Enhanced functionality of cantilever based mass sensors using higher modes," Applied Physics Letters, vol. 86, p. 233501, 2005.

[4] M. K. Ghatkesar, V. Barwich, T. Braun, J.-P. Ramseyer, C. Gerber, M. Hegner, H. P. Lang, U. Drechsler, and M. Despont, "Higher modes of vibration increase mass sensitivity in nanomechanical cantilevers," Nanotechnology, vol. 18, p. 445502, 2007.

[5] M. Domínguez, J. Pons-Nin, J. Ricart, A. Bermejo, and E. F. Costa, "A novel $\Sigma-\Delta$ pulsed digital oscillator (PDO) for MEMS," IEEE Sensors J., vol. 5, pp. 1379-1388, Dec. 2005.

[6] M. Domínguez, J. Pons-Nin, J. Ricart, A. Bermejo, E. F. Costa, and M. Morata, "Analysis of the $\Sigma-\Delta$ pulsed digital oscillator for MEMS," IEEE Trans. Circuits Syst. I, vol. 52, pp. 2286-2297, Nov. 2005.

[7] M. Domínguez, J. Pons-Nin, and J. Ricart, "General dynamics of pulsed digital oscillators," IEEE Trans. Circuits Syst. I, vol. 55, pp. 2038-2050, 2008.

[8] E. Blokhina, J. Pons, J. Ricart, O. Feely, and M. Domínguez, "Control of MEMS vibration modes with Pulsed Digital Oscillators: Part I Theory," IEEE Trans. Circuits Syst. I, vol. 57, no. 9, 2010.

[9] J. Ricart, J. Pons, E. Blokhina, S. Gorreta, J. Hernando, T. Manzaneque, J. L. Sanchez-Rojas, O. Feely, and M. Domínguez, "Control of MEMS vibration modes with Pulsed Digital Oscillators: Part II Simulation and experimental results," IEEE Trans. Circuits Syst. I, vol. 57, no. 9, 2010.

[10] K. Graff, Wave Motion in Elastic Solids. New York: Dover Publications Inc, 1975.

[11] A. H. Nayfeh, M. I. Younis, and E. M. Abdel-Rahman, "Reduced-order models for MEMS applications," Nonlinear Dynamics, vol. 41, pp. 211236, 2005.

[12] A. Teplinsky and O. Feely, "Limit cycles in a MEMS oscillator," IEEE Trans. Circuits Syst. II, vol. 55, pp. 882-886, Sept. 2008.

[13] E. Blokhina, O. Feely, and M. Dominguez, "Dynamics of the MEMS pulsed digital oscillator with multiple delays in the feedback loop," in In proc. of IEEE International Symposium on Circuits and Systems (ISCAS) 2009, Taipei, Taiwan, May, 24-27 2009. 